\title{
The effect of cyclic thermal stresses in the life of PET shrink's heating body
}

\author{
József Lakner, György Györök \\ Regional Educational and Innovation Center \\ Óbuda University \\ Budai Str. 45, H-8000 Székesfehérvár \\ lakner.jozsef@bmf.roik.hu \\ gyorok.gyorgy@bmf.roik.hu
}

\begin{abstract}
An automatic device carries out the size-reducing shrinking of polyethylene (PET) bottles using a heating technology featuring the "shape memory" polyethylene. In the device a lever pushes the bottle in the furnace at a temperature of $800-1300^{\circ} \mathrm{C}$ then it presses and shrinks it. We have a dual aim when examining the furnace. On one hand, our aim is to determine the relationship between weariness (the number of operation, time of operation) and electric resistance. Its practical function is to justify customer complaints via resistance measuring. Another purpose of our measurements is to offer such a controlling procedure that provides the longest life time under given circumstances in case of a given furnace.
\end{abstract}

\section{INTRODUCTION}

Fig. 1 and 2 show the environment of the measuring. For continual measuring we set a 40s switch-off and a 20s switchon time using a programmed microprocessor controller. The resistance measuring was realized after oligarchically changing cycles at 10, 20, 50, 100 etc. heating body cooled down to room temperature. Its actual operation conditions were simulated with minute cycle time, within that with a 20 second heating, during which its life time equaled 10000 cycles, and is equivalent of 55-hour long isotherm heat production. This is significantly smaller than life time measured without cycles. We constantly measured the resistance in both cases so as to understand the effect of the temperature cycles. By comparing the values we could make conclusions on the processes during the temperature change and on the cause of breakdown [5].

\section{RESULT OF MEASURING}

The change of resistance of heating body $(R)$ of cyclic heat load is shown in Fig.3. Significant fluctuations in the initial resistance of about one thousand cycles decreased, then increased approximately linearly. The measurement is completed 10.000 cycles, in practice it is expected lifetime of the heating body [1].

In isotherm case (continuous heating) the change of resistance of heating body can be seen in Fig.4. The resistance decreased rapidly for about 5 hours, then continued to decline but the rate of decline is much smaller and the destruction of the corresponding isothermal heat stress (55 hours) was stable. Furthermore, in contrast to the cyclic loading, the initial fluctuations are practically did not appear.

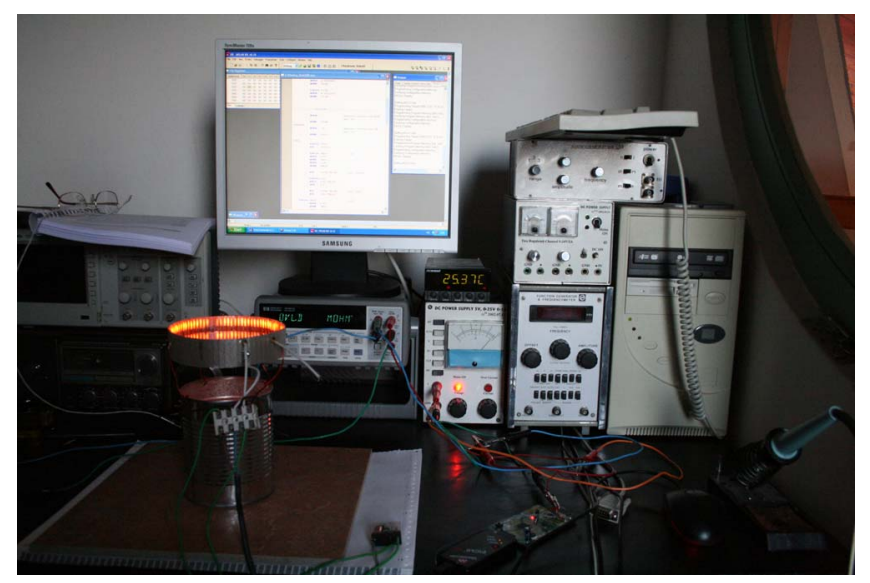

Fig. 1. The measuring system with the heating body switched on.

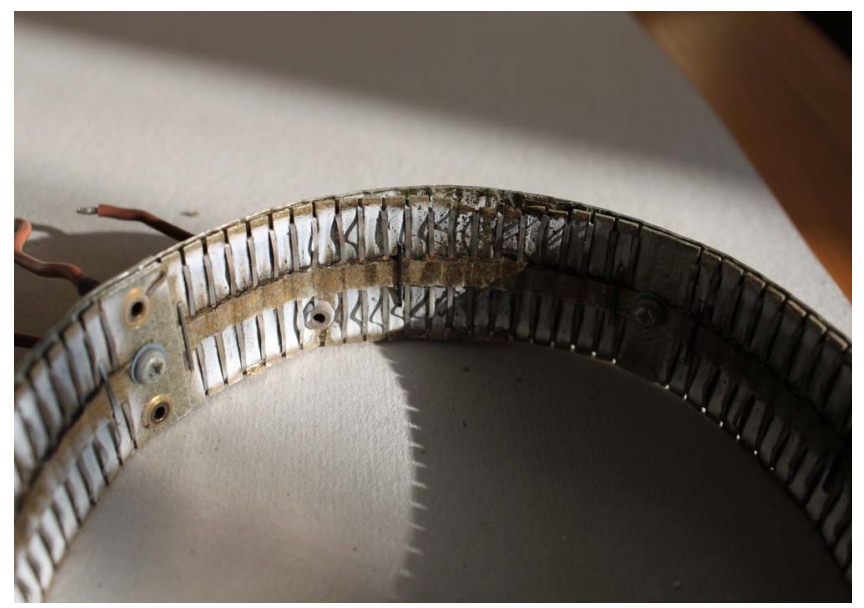

Fig. 2. Detail of the heating body, after the cyclical test.

\section{DisCUSSION}

The fluctuation of the resistance of the cyclical sample at the beginning of the measurement can be resulted in the change of the contact between the heating wire and the terminals as the sizes of the heating wire are changed by the periodical heating up and cooling and this can appear at the contacts as a series of small consecutive plastic deformations. These deformations have the joining of surfaces changed and by this 


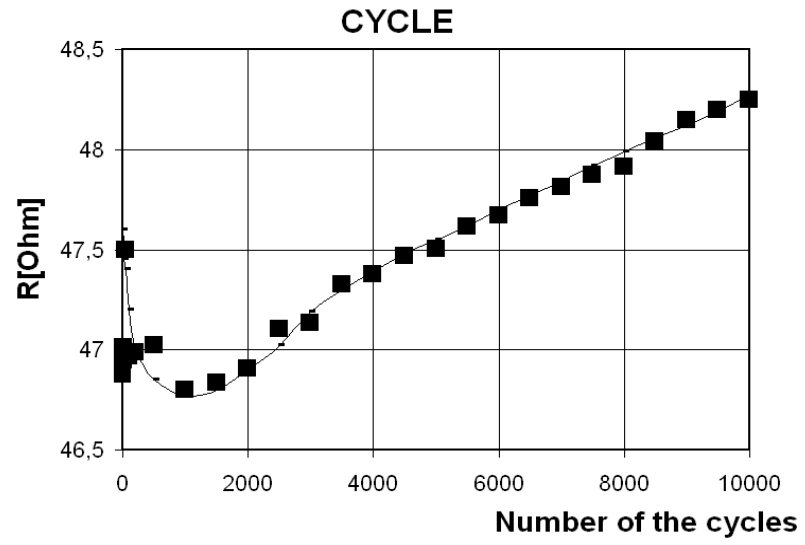

Fig. 3. The resistance change of the heating body, PET, as the function of the cycle number. The symbols are the measured data, the dashed line is the values given from elimination of the contact changes.

\section{ISOTHERM}

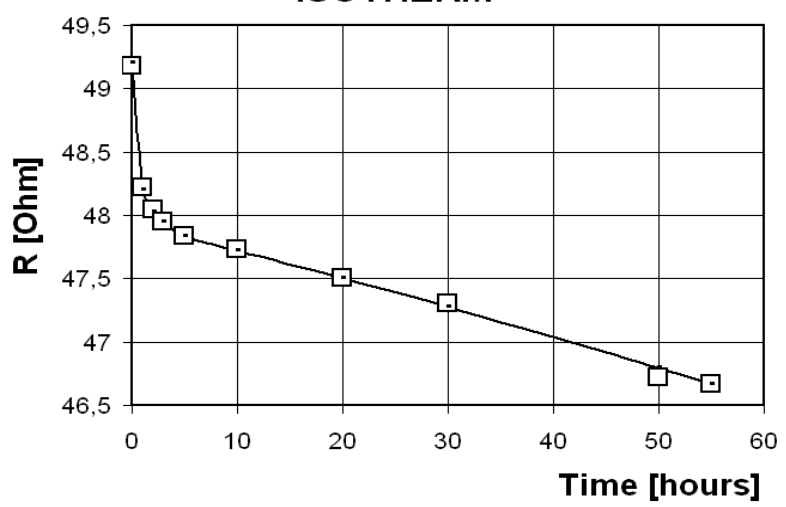

Fig. 4. The resistance change of the heating body, PET, as the function of the isotherm thermal load time. The symbols are the measured data, the dashed line is most probable values

means the contact resistance as well. After a number of cycles the contacts have been "welded" and from this time on they are not changed. Eliminating the change of resistance from the contacts the resistance decreases until about 1000 cycles and following the decrease it has began to increase ( Fig. 3).

In case of isotherm samples the resistance quickly decreases during the first five hours (Fig. 4). The cause of this resistance decrease can be the disappearance of the deformed structure of the heating wire (drawn wire) as in this time the dislocations have disappeared and the solid solution can be partly dissolved and a recovered-recrystallized structure has been formed, where all of the processes decrease the resistance [2].

The decrease of the resistance continued after 5 hours. As a bigger part of the resistance of the heating wire comes from the contribution of the alloying elements of concentration c, being in solid solution $\left(R_{c}\right.$ is the resistance contribution of unit concentration of alloys) which using the Mattiesen-rule will be

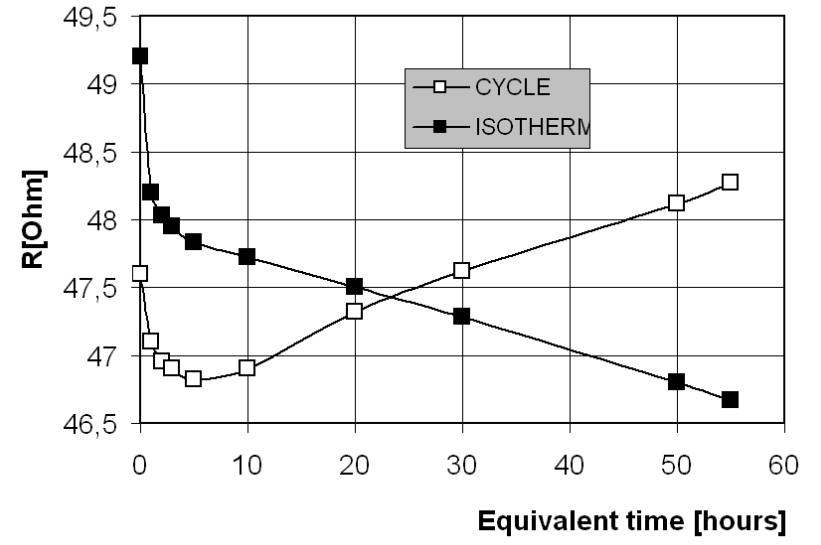

Fig. 5. The resistance change of the cyclical and isotherm samples as function of the time of equivalent thermal load.

$$
R=R_{M}+R_{c} c
$$

therefore its decrease can be connected with dissolution of the solid solution. The process goes on by diffusion, the rate of which as follows

$$
-\frac{d c}{d t}=D \cdot \operatorname{grad} \mathbf{c} \cong D \frac{\Delta c}{\lambda},
$$

where $D$ is the diffusion coefficient (depending on the temperature), $\lambda$ is the average diffusion distance and $\Delta c$ is the concentration difference within the diffusion distance [3]. As the process is rather slow (during 50 hours the chance of the resistance is smaller the $2 \%$, it means, that a few percent of the solid solution dissolved) therefore it can be a long distance diffusion process $\lambda>>0$, that is the precipitations have been formed on the grain borders, $\lambda \cong$ diameter of grain $/ 4$, or on the surface of wire, $\lambda \cong$ diameter of wire $/ 4$ (in this case including the oxidation and the evaporation on the surface). As the concentration is changed small, therefore $c \cong c_{0}$, where $c_{0}$ is the initial concentration, for this reason the $\Delta c$ concentration difference, is equal to the initial one, $\Delta c \cong \Delta c_{0}$, due to this the rate of the process using Eq.(2), is nearly constant. Comparing Eq.(1) and (2) the resistance the function of the time will be

$$
R=R_{0}-\alpha t
$$

where $R_{0}=R_{c} c_{0}$ and $\alpha=\frac{\Delta c}{\lambda}$ gives linear function (Fig. 4).

In order to compare the cyclical samples to the isotherm ones the equivalent thermal load time was calculated in the next

$$
t_{e q}=\frac{1}{180} n
$$

where $n$ number of cycle. 


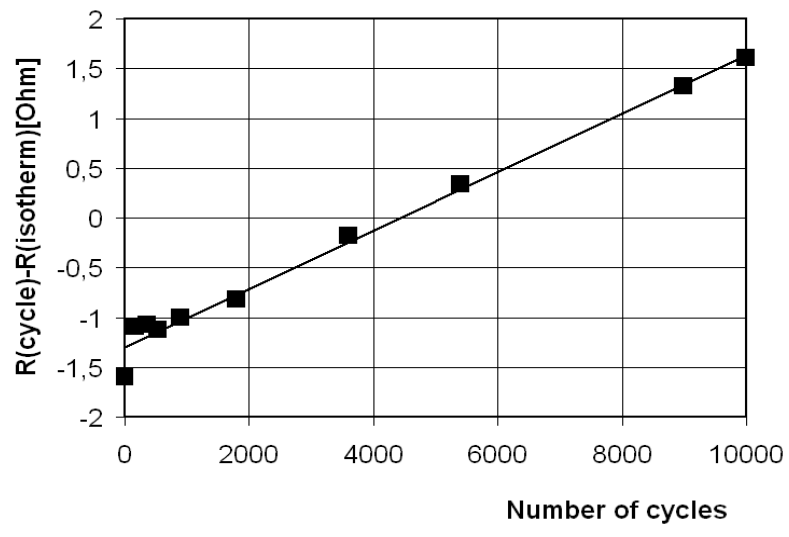

Fig. 6. The difference between resistances of the cyclical and isotherm samples as function of the time of equivalent thermal load.

The resistance changes have been described as the function of this time for both samples (Fig.5). The figure shows that the resistances of both samples decrease to 5 hours similarly, after this the resistance of the cyclical sample grows while the isotherm one decreases on.

The decrease of resistance of cyclical sample has turned over the increase after 5 hours. In this case as the processes mentioned above have carried out as well the increase of resistance is caused by the cyclical. Separating the latter the difference of the resistances between the two types of samples is imaged in Fig.6, which increase with equivalent thermal load time (the number of cycles) in direct proportion (The difference of resistance at null point has come from that of the two samples). As during the cyclical thermal load there are no processes related to the isotherm case, which would have resulted in plus resistance change, therefore the increase of resistance can be the consequence of the change (elongation) of the geometric form at the heat wire.

The geometrical shape of the heating wire is formed by three fixed points of every turns to an insulated body (Fig.7.a.). During heating in consequence of the difference between thermal expansion coefficients of the wire and the body the wire has bumped (Fig.7.b), while plastic deformation (creep) has also gone on, and after switching the heating off, during the cooling it has contracted, a pulling stress has been formed, under its influence the wire has elongated $l_{i}=l_{i-1}+\Delta l_{i}$ (Fig.7.c.) [1]. As a result of this at the end of the cycle the length of the wire has increased, its cross-section has decreased, $q_{i}=l_{i-1}-2 \Delta l_{i}$, and both together cause an increase of the resistance, namely

$$
\Delta R=R_{i}-R_{i-1} \cong R_{i-1} \frac{3 \Delta l_{i}}{l_{i}} .
$$

This process recurs cycle by cycle and the elongations, according to these the changes of resistance have been summarized. If the elongations of the cycles are about equal, $\Delta l_{i} \cong \Delta l$, and $\Delta l_{i}<<l$, in this case $l_{i} \cong l$ and $R_{i} \cong R_{0}$, then the resistance
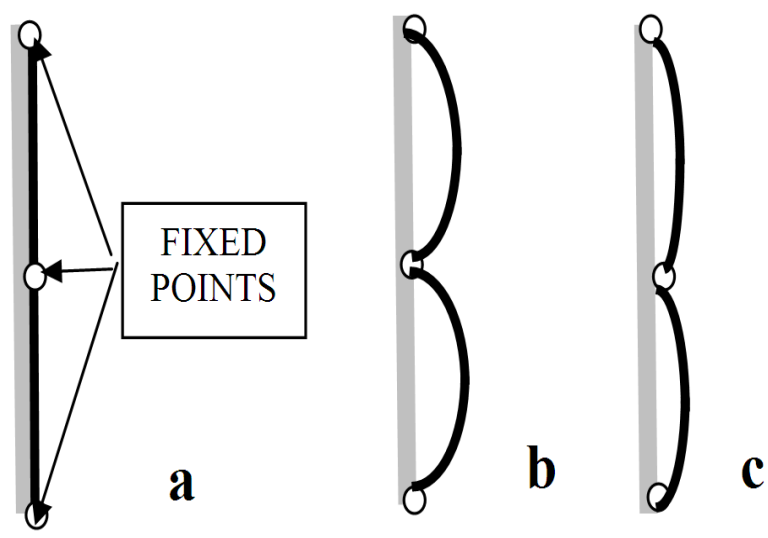

Fig. 7. The schematic figure of elongation of the heating wire during one cycle.

difference after $n$ cycles will be

$$
\Delta R_{n}=\sum_{i=1}^{n} R_{i-1} \frac{3 \Delta l_{i}}{l_{i}} \cong \beta n
$$

where $\beta=R_{0} \frac{\Delta l}{l}$ is the resistance contribution of one cycle. According to the Eq.(6) resistance difference,

$\Delta R_{n}=R($ cycle $)-R($ isotherm $)$ as the function of the number of cycles (equivalent thermal time) will be linear (Fig.6).

As a result of the cyclical load the material of the heating wire has fatigued, and has failure at a given number of cycles $\left(n_{M A X}\right)$, (fatigue limit). The mechanism of the failure is that the crack propagations being in the material have begun to grow by the cyclical stress and when they go over the whole cross section, the wire has fatigued [4]. In our case the fracture is speeded up by the local melting formed by the increased temperature caused by the increase of the resistance at this cross section. The crack propagations can appear at the so called grain sizes of three, thus the fatigue limit and the life time, of course, is the function of the recovered-recrystallized structure, which can be changed by the parameters of the softening temperature [4]. Recently these are the parameters of a spontaneous softening process carrying out during the first 1000 cycle.

\section{CONCLUSIONS AND PROPOSITIONS}

The fracture of the heating wire, PET can be connected to the fatigue caused by the cyclical load, therefore the possibilities for extension of life time are the next:

1) To choose a wire material having better fatigue parameters as used (e.g. Nikrokantal [1]).

2) To increase of fatigue limit by heat treatment of the wire (by the annealing before using).

3) To decrease the cycle number by delaying switch off. This can mean, that the equipment does not switch off between two bottles running after each other.

Solution of (2) requires further development, whereas (3) can be solved by control. 


\section{REFERENCES}

[1] Handbook. KANTHAL Appliance Heating Alloys Copyright by Kanthal $\mathrm{AB}$, Catalogue 1-A-2-3 02. 975000.

[2] Cahn, R. W. Recovery and Recrystallization, Physical Metallurgy. (Fourth Edition), 1996, p. 2399-2500.

[3] Doherty, R. D. Diffusive Phase Transformations in the Solid State, Physical Metallurgy. (Fourth Edition), 1996, p. 1363-1505.

[4] Thomson, R. M. Fracture, Physical Metallurgy. (Fourth Edition), 1996, p. $2207-2292$

[5] Gy. Györök, L. Simon Programozhat analg ramkr megszaktsos alkaImazsa mikrovezrl krnyezetben. IAlkalmazott informatika s hatrterletei Szimpzium, A Budapesti Mszaki Fiskola Regionlis Oktatsi s Innovcis Kzpont, 2009. november 6. Szkesfehrvr, ISBN ISBN 978-963-7154-94-2. 